\title{
Impact of proanthocyanidin-rich apple intake on gut microbiota composition and polyphenol metabolomic activity in healthy mildly hypercholesterolemic subjects
}

\author{
Athanasios Koutsos $^{1}$, Maria M. Ulaszewska ${ }^{2}$, Kajetan Trošt ${ }^{2,3}$, Jan Stanstrup ${ }^{2}$, Letizia Mariani ${ }^{2}$, \\ Amanda Galvin ${ }^{1}$, Tanya Braune ${ }^{1}$, Francesca Fava ${ }^{2}$, Elena Franciosi ${ }^{2}$, Massimo Pindo ${ }^{2}$, \\ Matthias Scholz, Fulvio Mattivi ${ }^{2,4}$, Kieran Tuohy ${ }^{2}$ and Julie Lovegrove ${ }^{1}$ \\ ${ }^{1}$ Hugh Sinclair Unit of Human Nutrition and the Institute for Cardiovascular and Metabolic Research (ICMR), \\ Department of Food and Nutritional Sciences, University of Reading, Reading, United Kingdom, \\ ${ }^{2}$ Research and Innovation Centre, Fondazione Edmund Mach, San Michele all'Adige, Italy, \\ ${ }^{3}$ Steno Diabetes Center, Copenhagen, Denmark and \\ ${ }^{4}$ University of Trento, Trento, Italy
}

\begin{abstract}
Apples are a rich source of polyphenols and fiber. Proanthocyanidins (PAs), the largest polyphenolic class in apples, can reach the colon almost intact where they interact with the gut microbiota producing simple phenolic acids. These metabolites have the potential to modulate gut microbiota composition and activity and impact on host physiology. A randomized, controlled, crossover, dietary intervention study was performed to determine the broad effects of whole apple intake on fecal gut microbiota composition and activity. Forty heathy mildly hypercholesterolemic volunteers ( 23 women, $17 \mathrm{men}$ ), with a mean BMI $\left( \pm\right.$ SD) $25.3 \pm 3.7 \mathrm{~kg} / \mathrm{m}^{2}$ and age $51 \pm 11$ years, consumed 2 apples/day (Renetta Canada, rich in PAs), or a sugar matched control apple beverage, for 8 weeks separated by a 4-week washout period in a random order. Fecal and 24-h urine samples were collected before and after each treatment. The broad effects of apple intake on fecal gut microbiota composition were explored by the high throughput sequencing (HTS) of 16S rRNA gene 1llumina MiSeq sequencing (V3-V4 region). Sequencing data analysis was performed using the Quantitative Insight Into Microbial Ecology (QIIME) open-source pipeline version 1.9.1. Specific bacterial groups were also enumerated using the quantitative Fluorescence In Situ Hybridization (FISH). Furthermore, the potential formation of microbial polyphenol metabolites, after apple intake, was explored in urine using Liquid Chromatography (LC) High-Resolution Mass Spectrometry (HRMS) metabolomics. Preliminary analysis showed no changes in gut microbiota abundances measured by Illumina MiSeq, after correction for multiple testing. Apple intake significantly decreased Enterobacteriaceae population $(P=0.04)$ compared to the control beverage, as determined with FISH. Twenty-four polyphenol microbial metabolites were identified in higher concentrations in the apple group $(P<0.05)$ compared to the control, including valerolactones, valeric and phenolic acids. In conclusion, preliminary data suggest that the daily intake of 2 Renetta Canada apples significantly decreased Enterobacteriaceae population, a family known for its pathogenic members, in healthy mildly hypercholesterolemic subjects. Moreover, several polyphenol microbial metabolites were identified, suggesting that microbial activity is crucial and a prerequisite for the absorption of apple polyphenols, producing active metabolites with potential health benefits.
\end{abstract}

\section{Conflict of Interest}

There is no conflict of interest 